\title{
Three Dimensional Numerical Study on the Efficiency of a Core-shell InGaN/GaN Multiple Quantum Well Nanowire LED
}

\author{
Chi-Kang Li \\ Graduate Institute of Photonics and Optoelectronics \\ and Department of Electrical Engineering, \\ National Taiwan University, Taipei, Taiwan, 10617.
}

\author{
Yuh-Renn Wu \\ Graduate Institute of Photonics and Optoelectronics \\ and Department of Electrical Engineering, \\ National Taiwan University, Taipei, Taiwan, 10617. \\ Email: yrwu@cc.ee.ntu.edu.tw
}

\begin{abstract}
This paper presents the development of 3D fully selfconsistent FEM Poisson and drift-diffusion solver. We use this solver to investigate the current flow in the coreshell multiple quantum well LED.. The current spreading effect was examined to determine the carrier distribution at the sidewall of core-shell nanowire LEDs. The results revealed that a larger aspect ratio by increasing the nanowire height could increase the nonpolaractive area volume and reduce the droop effect at the same current density. Making the current spreading length exceed a greater nanowire height is critical for reducing the droop effect. In addition, this paper presents a discussion on the influences of the spacing between each nanowire on corresponding nanowire diameters.
\end{abstract}

\section{INTRODUCTION}

GaN-based light-emitting diodes (LEDs) have become an important lighting source for energy saving, and are gradually replacing traditional lighting sources because of their high energy efficiency and longer device lifetime. However, the conventional planar GaN-based LED grown on a c-plane cannot avoid the quantum confined stark effect (QCSE) induced by the piezoelectric field. The defect or dislocation density of $\mathrm{GaN}$ materials caused by the lattice mismatch between $\mathrm{GaN}$ and sapphire is also relatively large in limiting the internal quantum efficiency (IQE). At the same time, as the nanotechnology has matured, the fabrication of LEDs with nanowire geometries has significantly progressed in making three dimensional (3D) LEDs feasible. The preferred structure of nanowire LEDs involves a core-shell geometry [1], which enhances the active area effectively. The largest advantage of core-shell nanowire LEDs (CNLs) is the nonpolar surface at the sidewall. Because the resistivity of the p-GaN layer is inherently high and the cross-sectional area for current flow is restricted within the core of the nanowire, maintaining the homogeneous carrier distribution might be the key factor for improving the efficiency of CNLs. To model these factors, as fully $3 \mathrm{D}$ program will be the better choice in the study.

\section{FORMALISM}

To simulate the CNL precisely, we used the GMSH program [2], which is a 3D grid generator based on the finite element method (FEM) to construct a 3D hexagonal nanowire and
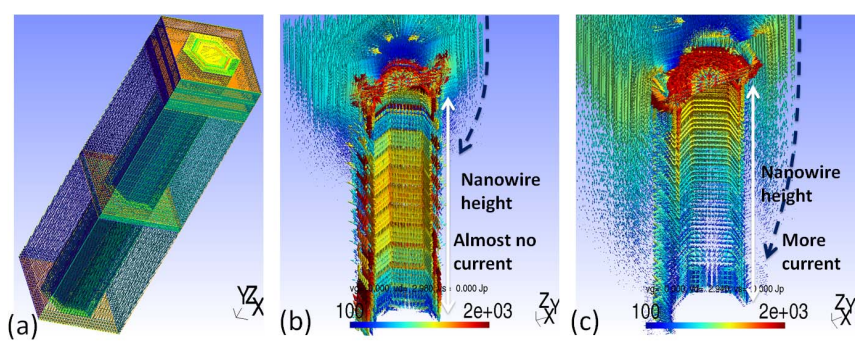

Fig. 1. (a) The simulation structure with mesh grids constructed by the GMSH program. (b) The cross-sectional (y-z plane) current vector plot of the CNL without TCL. (c) The cross-sectional (y-z plane) current vector plot of the CNL with TCL.

meshed it (Fig. 1(a)). Thereafter, the nonlinear 3D FEM Poisson and drift-diffusion equations are solved self-consistently to obtain the convergent profile. Figures 1(b) and 1(c) are the current vector plot for the CNL without and with TCL. This study used the Shockley-Read-Hall (SRH) model to account for defect-assisted recombination. The electron-hole separation due to QCSE and the carrier-screening effect due to carrier injection will be considered in the self-consistent Poisson and drift-diffusion solver. When the quantum confined effects of electron and hole wave are considered, the electronhole overlap should be better than the simulation result from the classical Poisson and drift-diffusion model. Therefore, the radiative recombination and Auger recombination will increase at the same time. In the c-plane QW, many numerical studies [3] used $50 \%$ or less theoretical polarization value to account the better overlapping and the strain relaxation effect. In our case, a $50 \%$ theoretical polarization value [4] was used in the c-plane QW. However, in this study, the active volume of the nonpolar plane is much larger than the c-plane QW. As we know, for the nonpolar-QW, the QCSE is weak and the classical model and quantum model all give $\sim 100 \%$ electronhole overlapping. Therefore, the overlapping in this study would not be a big issue because the active volume of the nonpolar plane QW is at least 10 times larger than the cplane QW, where the c-plane QW does not play a major role here. In this study, the nonradiative carrier lifetime $\tau_{n}$ and 
$\tau_{p}$ were $5.0 \times 10^{-8} \mathrm{~s}$. The radiative recombination coefficient $(B)$ was set at $2.0 \times 10^{-11} \mathrm{~cm}^{3} / \mathrm{s}$ and the Auger recombination coefficient $(C)$ was set at $2.0 \times 10^{-31} \mathrm{~cm}^{6} / \mathrm{s}$. These parameters are commonly used to examine droop effects [3]. The previous results based on 2D and 3D programs can be found in [5], [6].

\section{RESULT AND DISCUSSION}

The epi-layers of simulated structures are composed of a p-GaN layer, undoped 3-pair $\mathrm{In}_{0.14} \mathrm{Ga}_{0.86} \mathrm{~N} / \mathrm{GaN}$ multiple quantum wells (MQWs), and an n-GaN layer. In calculating the current density, we fixed the nanowire density in the same $600 \mathrm{~nm} \times 600 \mathrm{~nm}$ chip area to examine the device performance thoroughly in different situations. At first, we investigated the height of CNLs without the TCL to examine the current spreading effect in the vertical direction. Because of the low mobility and high activation energy of the p-type doping layer, the resistance is so large that it impedes the hole transport compared to the electron transport. Therefore, as Fig. 2(a) shows, in a CNL without a TCL, the carrier distribution in the vertical direction along nonpolar QWs is inhomogeneous, which leads to a non-uniform radiative recombination distribution. In addition, the improvement of the droop effect weakens (Fig. 2(b)) as the nanowire height increases because the current cannot reach the bottom for using nonpolar QWs completely. Hence, using a TCL or enhancing the hole layer conductivity is required for optimizing the current spreading effect in the vertical direction. As the height of a nanowire increases, the maximum resistivity limitation decreases as the inset of Fig. 2(c) shows. The findings indicate that a TCL with a resistivity equal to $1.25 \times 10^{-2} \Omega \cdot \mathrm{cm}$ would optimize the current spreading effect for $\mathrm{h}$ equal to $2.5 \mu \mathrm{m}$ as shown in Fig. 2(c). In addition, the findings establish that the optimized condition for current spreading is critical for mitigating the droop effect, especially for the higher nanowire height (Fig. 2(d)).

In this study, we demonstrated the difference between using a $2 \mathrm{D}$ and a $3 \mathrm{D}$ simulation. In the $2 \mathrm{D}$ simulation, when the rod density is fixed and the nanowire diameter increases with a lower aspect ratio, the IQE curve degrades slightly because of the enhancement of the polar QW area. However, the 3D simulation results a different result: the droop effect is reduced by a larger diameter with a lower aspect ratio. Because of the increase of the nanowire diameter in a real 3D problem, not only does the area of polar QWs increase, but the area of nonpolar QWs also increases at the same rate. We found there is a different current spreading effect between the 2D and $3 \mathrm{D}$ simulation because the device resistance for $2 \mathrm{D}$ and $3 \mathrm{D}$ simulation is different. Therefore, when using a 2D simulation, researchers should be aware of these effects, and that some modification might be needed in explaining the 2D simulation. As the nanowire diameter increases, the spacing between each nanowire shrinks. Hence, we could expect the current spreading to worsen, thus limiting the improvement of the droop effect.
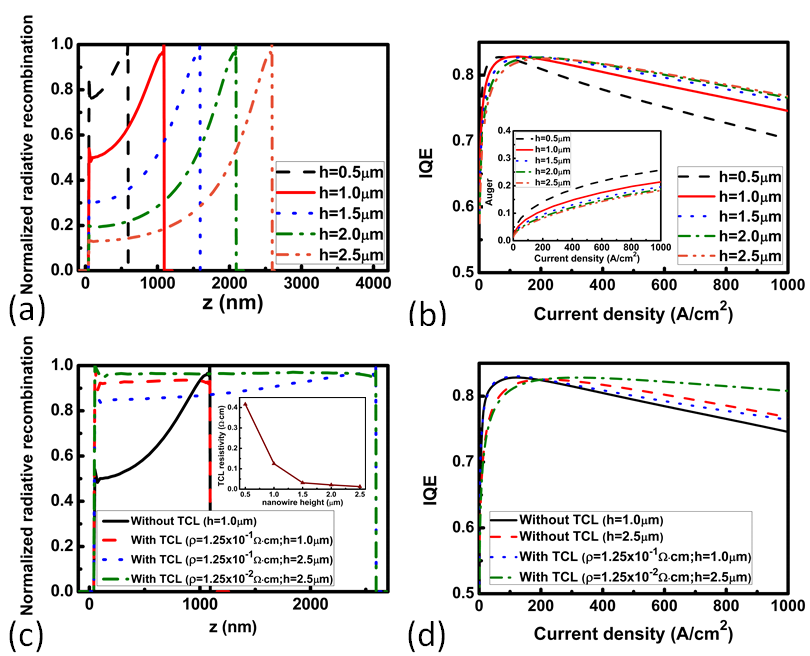

(b)

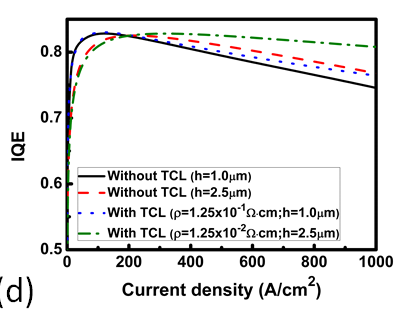

Fig. 2. (a) The vertical line plot of normalized radiative recombination along the outer nonpolar QW with various nanowire heights. (b) IQE versus different current densities $\left(\mathrm{A} / \mathrm{cm}^{2}\right)$ with various nanowire heights. The inset in (b) is the ratio of Auger recombination versus different current densities $\left(\mathrm{A} / \mathrm{cm}^{2}\right)$. (c) The vertical line plot of normalized radiative recombination along the outer nonpolar QW for the optimized current spreading effect. The inset in (c) is the required resistivity of the TCL to optimize the current spreading effect with different nanowire heights. (d) IQE versus different current densities $\left(\mathrm{A} / \mathrm{cm}^{2}\right)$ for the optimized current spreading effect.

\section{CONCLUSION}

This paper presents a 3D numerical analysis of the current spreading effect and efficiency droop in CNLs. With a fully self-consistent 3D simulator, it is possible to optimize the current spreading. The suggested maximum resistivity of different nanowire heights was calculated. With the increase of the nanowire height, the onset current of the droop effect is delayed and the efficiency degradation decreases. As the nanowire diameter increases, both the polar and nonpolar QW areas increase. However, nonpolar QWs dominate the droop effect; hence, the reduction of the carrier density inside the active region mitigates the droop effect.

\section{REFERENCES}

[1] R. Koester, J.-S. Hwang, D. Salomon, X. Chen, C. Bougerol, J.-P. Barnes, D. L. S. Dang, L. Rigutti, A. d. L. Bugallo, G. Jacopin, M. Tchernycheva, C. Durand, and J. Eymery, "M-Plane Core-Shell InGaN/GaN MultipleQuantum-Wells on GaN Wires for Electroluminescent Devices," Nano Lett., vol. 11, no. 11, pp. 4839-4845, 2011.

[2] C. Geuzaine and J. F. Remacle, "Gmsh: A 3-D finite element mesh generator with built-in pre- and post-processing facilities," Int. J. Numer. Meth. Eng., vol. 79, no. 11, pp. 1309-1331, 2009.

[3] J. Piprek, "Efficiency droop in nitride-based light-emitting diodes," Phys. Stat. Sol. (A), vol. 207, no. 10, pp. 2217-2225, 2010.

[4] V. Fiorentini, F. Bernardini, and O. Ambacher, "Evidence for nonlinear macroscopic polarization in III-V nitride alloy heterostructures," Appl. Phys. Lett., vol. 80, no. 7, pp. 1204-1206, 2002.

[5] C.-K. Li and Y.-R. Wu, "Study on the Current Spreading Effect and Light Extraction Enhancement of Vertical GaN/InGaN LEDs," IEEE Trans. Electron Devices, vol. 59, no. 2, pp. 400-407, 2012.

[6] J.-W. Yu, C.-K. Li, C.-Y. Chen, Y.-R. Wu, L.-J. Chou, and L.-H. Peng, "Transport properties of gallium nitride nanowire metal-oxidesemiconductor transistor," Appl. Phys. Lett., vol. 99, no. 15, p. 152108 , 2011 . 